\title{
RECEIVED
}

\section{JUN 114993}

OSTI

DOE/ER/40561-101-INT92-08-03

\section{Strange Hadronic Matter}

Jürgen Schaffner1,2, Carl B. Dover1,3, Avraham Gal1,2,4, Carsten Greiner 1,5, and Horst Stöcker1,2

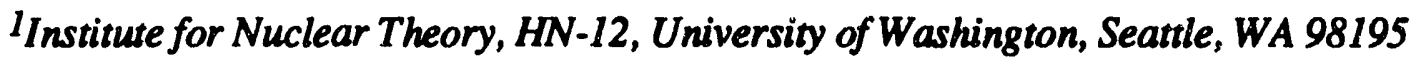

2Institute für Theoretische Physik, J.W. Goethe Universität, Frankfurt, GERMANY

3 Brookhaven National Laboratory, Upton, Ny 11973

${ }^{4}$ Racah Institute of Physics, The Hebrew University, Jerusalem 91904, ISRAEL

5Department of Physics, Duke University, Durham, NC 27706

\section{PREPARED FOR THE U.S. DEPARTMENT OF ENERGY UNDER GRANT DE-FG06-90ER40561}

This report was prepared as an account of work sponsored by the United States Government. Neither the United States nor any agency thereof, nor any of their employees, makes any warranty, express or implied, or assumes any legal liability or responsibility for the accuracy, completeness, or usefulness of any information, apparatus, product, or process disclosed, or represents that its use would not infringe privately owned rights. Reference herein to any specific commercial product, process, or service by trade name, mark, manufacturer, or otherwise, does not necessarily constitute or imply its endorsement, recommendation, or favoring by the United States Government or any agency thereof. The views and opinions of authors expressed herein do not necessarily state or reflect those of the United States Government or any agency thereof. 


\title{
STRANGE HADRONIC MATTER
}

\author{
Jürgen Schaffner ${ }^{1,2}$, Carl B. Dover ${ }^{1,3}$, Avraham Gal ${ }^{1,2,4}$,
}

\section{Carsten Greiner ${ }^{1,5}$ and Horst Stöcker ${ }^{1,2}$}

1) National Institute for Nuclear Theory, University of Washington, Seattle, WA 98195

2) Institut für Theoretische Physik, J.W. Goethe Universität, Frankfurt, Germany

3) Brookhaven National Laboratory, Upton, NY 11973

4) Racah Institute of Physics, The Hebrew University, Jerusalem 91904, Israel

5) Department of Physics, Duke University, Durham, NC 27706

\begin{abstract}
In an extended mean field theory, we find a large class of bound multistrange objects, formed from combinations of $\left\{p, n, \Lambda, \Xi^{0}, \Xi^{-}\right\}$baryons, which are stable against strong decay. We predict a maximal binding energy per baryon of $E_{B} / A \approx-21 \mathrm{MeV}$, strangeness per baryon $f_{s} \approx 1-1.2$, charge per baryon $f_{q} \approx-0.1$ to 0.1 , and baryon density 2.5-3 times that of ordinary nuclei. For $A \geq 6$, we obtain stable combinations involving only $\left\{\Lambda, \Xi^{0}, \Xi^{-}\right\}$hyperons.
\end{abstract}

This manuscript has been authored under contract number DE-AC02-76-CH00016 with the U.S. Department of Energy. Accordingly, the U.S. Government retains a non-exclusive, royalty-free license to publish or reproduce the published form of this contribution, or allow others to do so, for U.S. Government purposes. 
Strangeness $(S)$ remains a largely unexplored degree of freedom in strongly interacting baryonic matter. In addition to ordinary nuclei $(S=0)$, there exists a modest body of data on $S=-1,-2$ states involving one or two $\Lambda$ hyperons bound to a nuclear core ${ }^{1}$, but there is no information available on systems with $S \leq-3$. It has been speculated ${ }^{2,3}$ that states of quark matter ("strangelets") with large strangeness per baryon ( $S \mid / A \sim 1)$ might be even more stable than normal nuclei. The existence of stable strangelets could have significant ramifications ${ }^{4}$, for instance in astrophysics (nucleosynthesis, cores of neutron stars) and for relativistic heavy ion collisions, where the production of both stable and metastable objects might be enhanced if a transition to quark-gluon plasma takes place ${ }^{5}$.

Strangelets ${ }^{2,3}$ are hypothetical objects in which the quarks $(u, d, s$ in approximately equal numbers) are confined to a single large bag. In this paper, we consider bound states of hyperons $\left(\Lambda, \Xi^{0}, \Xi^{-}\right)$and nucleons $(n, p)$, where the binding arises from attractive mean fields. In this case, the strange quarks are localized within individual hyperons, which are assumed to retain their identity in the bound system. Hyperons are distinguishable from nucleons, and can occupy shell model states of the same orbital angular momentum $\ell$ and total spin $j$ as nucleons. Using an extension of the relativistic mean field (RMF) theory ${ }^{6-8}$, we form a succession of multi-strange nuclei by occupying the single particle bound states supported by the mean field. Remarkably, we obtain stable systems which display many of the features of hypothetical strange quark matter (large strangeness fraction $f_{s}=|S| / A \sim$ $0.6-1.2$, small net charge fraction $f_{q}=q / A \sim 0.1$ to -0.1 and densities $\rho \sim(2-3) \rho_{0}$, where $\rho_{0} \simeq 0.16 \mathrm{fm}^{-3}$ is the density of non-strange nuclear matter). However, the binding energy per particle $E_{B} / A$ for multi-strange nuclei, limited by the single particle well depths, is of order -10 to $-20 \mathrm{MeV}$, much less than the $\Lambda-N$ mass difference of $175 \mathrm{MeV}$. Thus these objects, while erijoying stability against strong decay, will decay by weak interactions with a typical lifetime $\tau \sim 10^{-10}$ sec or less. Strangelets, on the other hand, could conceivably have much larger binding energy, and also be stabilized against weak decay.

Several earlier calculations ${ }^{7-11}$ of strange hadronic matter (SHM) have focused on mixtures of $\Lambda$ hyperons and nucleons, although the rôle of the heavier $\Sigma$ and $\Xi$ hyperons in the equation of state of neutron stars has been explored ${ }^{12,13}$. In RMF calculations s, $^{7,10}$ with $\Lambda$ 's and nucleons, a variety of multi- $\Lambda$ hypernuclei were found, with binding per baryon as large as $E_{B} / A \approx-3 \mathrm{MeV}$, and $f_{s} \leq 0.2$. An example is shown in Fig. 1 for a 
${ }^{56} \mathrm{Ni}$ nuclear core plus $n_{\Lambda} \Lambda$ hyperons, using a standard RMF calculation with mean ficlds generated by scalar $(\sigma)$ and vector $(\omega)$ meson exchange ${ }^{6}$. The filled circles correspond to closed $\Lambda$ shells with $n_{\Lambda}=0,2,8,14$. We have noted a key point, missed in previous work, that for $n_{\Lambda}$ greater than some critical number, a system of $N$ 's and $\Lambda$ 's collapses via the reactions

$$
\Lambda+\Lambda \rightarrow \Xi^{-}+p, \quad \Xi^{0}+n
$$

Because of binding effects, reaction (1) can induce a strong decay of the many-body system, even though the reverse reactions $\Xi N \rightarrow \Lambda \Lambda$ proceed with sizable energy release $Q=28.3,23.2 \mathrm{MeV}$, respectively, in free space. Therefore, stable SHM necessarily includes a mixture of $\Lambda$ 's and $\Xi$ 's, such that the decays of Eq. (1) are Pauli-blocked ${ }^{14}$ in both directions. Note that systems with $\Sigma$ 's are generally not stable in RMF calculations, since the substantial energy release $\uparrow \approx 75-80 \mathrm{MeV}$ in reactions like $\Sigma N \rightarrow \Lambda N$ or $\Sigma \Xi \rightarrow \Lambda \Xi$ cannot be overcome by binding effects. Returning to Fig. 1, we emphasize the open circles and squares, which correspond to stable systems with $n \Xi \neq 0$. Adding $\Xi$ 's to the $A=64$, $n_{\Lambda}=8$ configuration leads to a stable sequence shown as open circles for $n_{\Xi}=2,4,10$. Similarly, adding $\Xi$ 's to the $A=70, n_{\Lambda}=14$ system yields a sequence (open squares) which extends to $n_{\Xi^{0}}=18, n_{\Xi^{-}}=8$, with $E_{B} / A=-12.3 \mathrm{MeV}, f_{s}=0.69, f_{q}=0.21$. For the next closed $\Lambda$ shell, at $n_{\Lambda}=18$, our calculation for $A=74$ yields no stable $\left\{{ }^{56} \mathrm{Ni}+18 \Lambda\right\}$ configuration, since the processes (1) destabilize it. Fig. 1 does show two stable states for $A=74$, with $\left(n_{\Lambda}, n_{\Xi^{0}}, n_{\Xi^{-}}\right)=(8,8,2)$ and $(14,2,2)$, which do not have the same charge and strangeness as the $(18,0,0)$ configuration. We emphasize that all of the configurations depicted in Fig. 1 are stable with respect to any of the $\Xi N \leftrightarrow \Lambda \Lambda$ reactions.

We now describe briefly the essence of our RMF calculations, relegating the details to Ref. 15. We consider two models, one with a weak and another with a strong hyperonhyperon $(Y Y)$ interaction, denoted as Models 1 and 2, respectively. The latter is more in line with the strongly attractive $\Lambda \Lambda{ }^{1} S_{0}$ interaction suggested by the few available $\Lambda \Lambda$ hypernuclear events ${ }^{16}$. In Model 1 , we simply add the $\left\{\Xi^{-}, \Xi^{0}\right\}$ isospin $1 / 2$ doublet, coupled to the usual $\sigma$ and $\omega$ isoscalar fields and to the isovector $\rho$ field. For the $\omega$ coupling constants $g$, we use ratios $g_{\omega} \equiv=g_{\omega \Lambda} / 2=g_{\omega N} / 3$ given by the constituent quark model (SU(6) symmetry). The $\sigma$ couplings are then determined by fitting the $\Lambda$ and $\equiv$ well depths ${ }^{1,17}$ in nucleon matter, $U_{\Lambda}^{(N)} \approx 30 \mathrm{MeV}$ and $U_{\Xi}^{(N)} \approx 28 \mathrm{MeV}$. Model 1, which 
exhibits rather weak $Y Y$ interactions, nevertheless implies the existence of stable SHM over a wide range of $\left\{n_{p}, n_{n}, n_{\Lambda}, n_{\Xi^{0}}, n_{\Xi-}\right\}$, typically with large strangeness $\left(f_{s} \sim 0.6\right)$, small charge $\left(f_{q} \sim 0.1\right)$ and maximum binding energies of order $E_{B} / A \sim-13 \mathrm{MeV}$. The small net charge is due to the tendency of $\Xi^{-}$'s and protons to occupy similar orbits so as to minimize the total Coulomb repulsion. This renders it possible for SHM to be stable in the bulk limit $(A \rightarrow \infty)$, because fission does not occur for large $A$ as in ordinary nuclei.

In Model 2, we incorporate stronger $Y Y$ interactions, as suggested by the sparse $\Lambda \Lambda$ data $^{16}$ and also by a one-boson exchange model ${ }^{18}$ consistent with the $N N, \Lambda N$ and $\Sigma N$ scattering data. We estimate, based on this information, and the value $U_{N}^{(N)} \approx 80 \mathrm{MeV}$ from standard RMF calculations ${ }^{6,7}$, potential depths

$$
U_{\Lambda}^{(\Xi)} \approx U_{\Xi}^{(\Xi)} \approx 2 U_{\Lambda}^{(\Lambda)} \approx 40 \mathrm{MeV}
$$

for hyperons embedded in hyperon matter at $\rho \Xi \approx \rho_{0}$ and $\rho_{\Lambda} \approx \rho_{0} / 2$. In order to obtain the necessary attraction, we supplement the $\sigma+\omega$ model by a second scalar-vector pair $\sigma^{*}$ and $\phi$ of isoscalar meson fields, which are assumed to couple only to hyperons. For the $\phi(1020)$ couplings, we use the quark model relationships $g_{\phi \Xi}=2 g_{\phi \Lambda}=-2 \sqrt{2} g_{\omega N} / 3$. For the $\sigma^{*}$, we use the mass of the observed $f_{0}(975)$ meson, but treat its couplings purely phenomenologically so as to satisfy Eq. (2). This yielded $g_{\sigma^{*} \Lambda} / g_{\sigma N}=0.69, g_{\sigma^{*}} \Xi / g_{\sigma N}=$ 1.25. We remark that although the $Y Y$ interaction has been appreciably strengthened in Model 2 with respect to Model 1, we are still somewhat short of reproducing the binding energy ${ }^{16}$ of the light system ${ }_{\Lambda \Lambda}^{6} \mathrm{He}$.

We have investigated Models 1 and 2 for a wide variety of SHM configurations based on various doubly magic nuclear cores. Representative results are shown in Figs. 2 and 3 , each of which displays three families of stable sequences. The jagged nature of these plots is due to the effects of shell closures, which are superimposed on a smooth $A, f_{s}$, or $f_{q}$ dependence which may be represented by a generalization of the familiar BetheWeizsäcker mass formula for nuclei. There is an approximately linear correlation between $f_{s}$ and $f_{q}$, rather similar to the relation $f_{q}=\left(1-f_{s}\right) / 2$ characteristic of isospin saturated strange quark matter. The approximate parabolic behavior evident in Figs. 2 and 3 near the minima can be parametrized as

$$
\frac{E_{B}}{A} \simeq\left(\frac{E_{B}}{A}\right)_{\min }+c_{s}\left(f_{s}-f_{s}^{\min }\right)^{2} \simeq\left(\frac{E_{B}}{A}\right)_{\min }+c_{q}\left(f_{q}-f_{q}^{\min }\right)^{2}
$$


One family of points in Figs. 2 and 3 gives $E_{B} / A$ for Model 2 sequences based on ${ }^{56} \mathrm{Ni}$ $(N=Z=28)$ and ${ }^{180} \mathrm{Th}(N=Z=90)$ cores, as a function of $f_{s}$ (Fig. 2) or $f_{q}$ (Fig. 3). We note that $E_{B} / A$ approaches values as large as $\left(E_{B} / A\right)_{\min } \approx-21 \mathrm{MeV}$, for $f_{s}^{\min } \simeq 1.08$, $f_{q}^{\min } \simeq-0.04$, corresponding to systems with more hyperons than nucleons. The ${ }^{56} \mathrm{Ni}$ sequence starts from $f_{s}=0, f_{q}=0.5$, corresponding to a stable core, and extends to systems as large as $A \sim 300$. In contrast, the unstable ${ }^{180} \mathrm{Th}$ core requires a large injection of strangeness to stabilize it against baryon emission. In this case, the region of stability extends from $A \simeq 390\left(f_{s} \simeq 0.9, f_{q} \simeq 0.08\right)$ to $A \sim 625$.

Another family in Figs. 2 and 3 consists of sequences of stable SHM in Model 1, for ${ }^{132} \mathrm{Sn}(N=82, Z=50),{ }^{208} \mathrm{~Pb}(N=126, Z=82)$ and ${ }^{310} \mathrm{G}(N=184, Z=126)$ cores. Here, due to the weaker $Y Y$ interactions, $E_{B} / A$ achieves values only as large as $-13 \mathrm{MeV}$, with a correspondingly smaller strangeness content $\left(f_{s}^{\min } \sim 0.6\right)$ and a positive charge $\left(f_{q}^{\min } \sim 0.1\right)$. A more detailed breakdown of the ${ }^{56} \mathrm{Ni}$ sequence for Model 1 is shown in Fig. 1.

A third family of objects is seen as a cluster in the upper right-hand corner of Fig. 2 and the upper left of Fig. 3. These represent stable aggregates of purely hyperonic $\left\{\Lambda, \Xi^{0}, \Xi^{-}\right\}$ matter which occur in Model 2 but not in Model 1. For such systerns, we find $E_{B} / A$ as large as $-9 \mathrm{MeV}$, for $f_{s} \simeq 1.8$ and $f_{q} \simeq-0.3 \pm 0.1$. It is unlikely that such clusters are bound with only one or two species of hyperons. The lightest stable object of this type is likely to be $\left(2 \Lambda+2 \Xi^{0}+2 \Xi^{-}\right)$. Purely hyperonic matter, in contrast to SHM in general, is not stable in the bulk limit, because of the Coulomb repulsion generated by the $\Xi^{-}$'s.

In summary, we have introduced a strong hyperon-hyperon interaction into the relativistic mean field model, and explored its consequences for the stability of strange hadronic matter. We find a vast array of stable objects composed of $\left\{n, p, \Lambda, \Xi^{0}, \Xi^{-}\right\}$baryons, of arbitrarily large baryon number $A$, very high strangeness content $\left(f_{s}>1\right)$ and small net charge (even $f_{q}<0$ ). Even if the $Y Y$ interactions are weakened, as in Model 1, these features remain qualitatively valid. The existence of such bound states, and the possibility of stability in the bulk matter limit, may have important consequences for the disposition of the numerous strange particles produced in relativistic heavy ion collisions ${ }^{19}$, the strangeness distillation mechanism ${ }^{5}$ for the hadronization of quark-gluon-plasma, and the equation of state of neutron stars at high density ${ }^{11-13}$. These issues remain to be explored. 
It may be possible to produce some of the lightest metastable objects envisaged here in the laboratory, by means of high energy heavy ion collisions. Estimates based on a coalescence mechanism $^{20}$ yield very small production rates, for instance $3 \times 10^{-9}$ events per central $\mathrm{Au}+\mathrm{Au}$ collisions at $11.7 \mathrm{GeV} / \mathrm{c}$ for the lightest bound $\Xi$ system $\Xi^{0} \Lambda \Lambda^{7} \mathrm{He}$. However, a considerable enhancement of the rate may occur if quark gluon plasma is formed ${ }^{5,21}$.

\section{Acknowledgments}

We would like to thank Wick Haxton and the other members of the National Institute for Nuclear Theory at the University of Washington for their hospitality during our visits to Seattle. This collaborative effort was initiated during Program 8 at the Institute. One of us $(A G)$ was partially supported by an award of the Alexander von Humboldt Foundation and by the Basic Research Foundation of the Israel Academy of Sciences and Humanities. Another (CG) is supported by a Feodor Lynen fellowship of the AvH-Stiftung. The efforts of CG, JS and HS were supported in part by the Gesellschaft für Schwerionenforschung, the Deutsche Forschungsgemeinschaft via Graduiertenkolleg Theoretische und Experimentelle Schwerionenphysik, and the Bundesministerium für Forschung und Technologie. This manuscript has been authored under contract number DE-AC02-76CH00016 with the U.S. Department of Energy. 


\section{References}

1. R.E. Chrien and C.B. Dover, Ann. Rev. Nucl. Part. Sci. $\underline{39}$, 113 (1989).

2. S.A. Chin and A.K. Kerman, Phys. Rev. Lett. $\underline{43}, 1292$ (1979);

E. Witten, Phys. Rev. D30, 272 (1984).

3. E. Farhi and R.L. Jaffe, Phys. Rev. D30, 2379 (1984) and D32, 2452 (1985).

4. Proceedings of the International Workshop on Strange Quark Matter in Physics and Astrophysics, Eds. J. Madsen and P. Haensel, Nucl. Phys. 24B (Proc. Suppl.) 1-299 (1991).

5. C. Greiner, D.H. Rischke, P. Koch and H. Stöcker, Phys. Rev. D38, 2797 (1988);

C. Greiner and H. Stöcker, Phys. Rev. D44, 3517 (1992).

6. B.D. Serot and J.D. Walecka, Adv. in Nucl. Phys. $\underline{16}, 1$ (1986).

7. M. Rufa, J. Schaffner, J. Maruhn, H. Stöcker, W. Greiner and P.-G. Reinhard, Phys. Rev. $\underline{\text { C42, }} 2469$ (1990).

8. J. Schaffner, C. Greiner and H. Stöcker, Phys. Rev. 46, 322 (1992).

9. M. Rufa, H. Stöcker, J. Maruhn, P.-G. Reinhard and W. Greiner, J. Phys. G13, 143 (1987).

10. J. Mares and J. Žofka, Z. Phys. $\underline{\text { A333, }} 209$ (1989).

11. H.A. Bethe, G.E. Brown and J. Cooperstein, Nucl. Phys. $\underline{\text { A462, }} 791$ (1987).

12. J. Ellis, J.I. Kapusta and K.A. Olive, Nucl. Phys. $\underline{B 348}, 345$ (1991).

13. N.K. Glendenning, F. Weber and S.A. Moszkowski, Phys. Rev. $\underline{\text { C45 }}, 844$ (1992).

14. The possibility of stabilizing $\Xi ' s$ in light systems by Pauli blocking was discussed in Ref. 8. We find that the lightest stable system including a $\Xi$ is $\Xi^{0} \Lambda \Lambda^{7} \mathrm{He}\left({ }^{4} \mathrm{He}+2 \Lambda+\Xi^{0}\right)$.

15. J. Schaffner, C.B. Dover, A. Gal, D.I. Millener, and H. Stöcker, to be submitted to Ann. Phys.

16. R.H. Dalitz, D.H. Davis, P.H. Fowler, A. Montwill, J. Pniewski and J.A. Zakrzewski, Proc. R. Soc. London A426, 1 (1989);

C.B. Dover, D.J. Millener, A. Gal and D.H. Davis, Phys. Rev. C44, 1905 (1991).

17. C.B. Dover and A. Gal, Ann. Phys. 146, 309 (1983). 
18. M.M. Nagels, T.A. Rijken and J.J. deSwart, Phys. Rev. D12, 744 (1975) and D15, 2547 (1977). These papers define Model D, in which the ${ }^{1} \mathrm{~S}_{0} Y Y$ interactions are all comparable and strongly attractive.

19. O. Hansen, Comments on Nucl. and Part. Phys. 20, 1 (1992).

20. C.B. Dover, A.J. Baltz, S.H. Kahana, Yang Pang and T. Schlagel, Proceedings of the Workshop on Heavy on Physics at the AGS (HIPAGS), MIT, January, 1993 (in press).

21. H. Crawford, M. Desai and G.L. Shaw, Phys. Rev. D45, 857 (1992). 


\section{Figure Captions}

Fig. 1: Binding energy per baryon $E_{B} / A$, for Model 1 (weak $Y Y$ interaction) as a function of baryon number $A$, for bound states of strange hadronic matter consisting of a ${ }^{56} \mathrm{Ni}$ nuclear core plus differing numbers of $\Lambda, \Xi^{0}$ and $\Xi^{-}$ hyperons. The solid circles represent systems consisting only of the ${ }^{56} \mathrm{Ni}$ core plus $\Lambda$ 's, with $n_{\Lambda}=0,2,8,14$. The open circles correspond to objects with $n_{\Lambda}=8$, to which $\Xi$ hyperons are added $(n \Xi=2,4,10)$. These are more deeply bound than systems of the same $A$ which have $n_{\Lambda}>8, n \equiv=0$. The open squares have $n_{\Lambda}=14$, with $n \equiv=2,4,8,10,16,22,26$.

Fig. 2: Binding energy per baryon $E_{B} / A$ as a function of strangeness fraction $f_{s}$ for several sequences of SHM based on various nuclear cores as indicated. The super-heavy core ${ }^{310} \mathrm{G}$ has $N=184, Z=126$, and the symbol $Y$ refers to multi-hyperon systems containing no nucleons $(N=Z=0)$. The calculations for the ${ }^{132} \mathrm{Sn},{ }^{208} \mathrm{~Pb}$ and ${ }^{310} \mathrm{G}$ cores use Model 1 (weak $Y Y$ interaction), while those for ${ }^{56} \mathrm{Ni},{ }^{180} \mathrm{Th}$ and $Y$ use Model 2 (strong $Y Y$ interaction).

Fig. 3: $\quad E_{B} / A$ as a function of charge fraction $f_{q}$ for various sequences of stable SHM. The labeling is the same as in Fig. 2. 


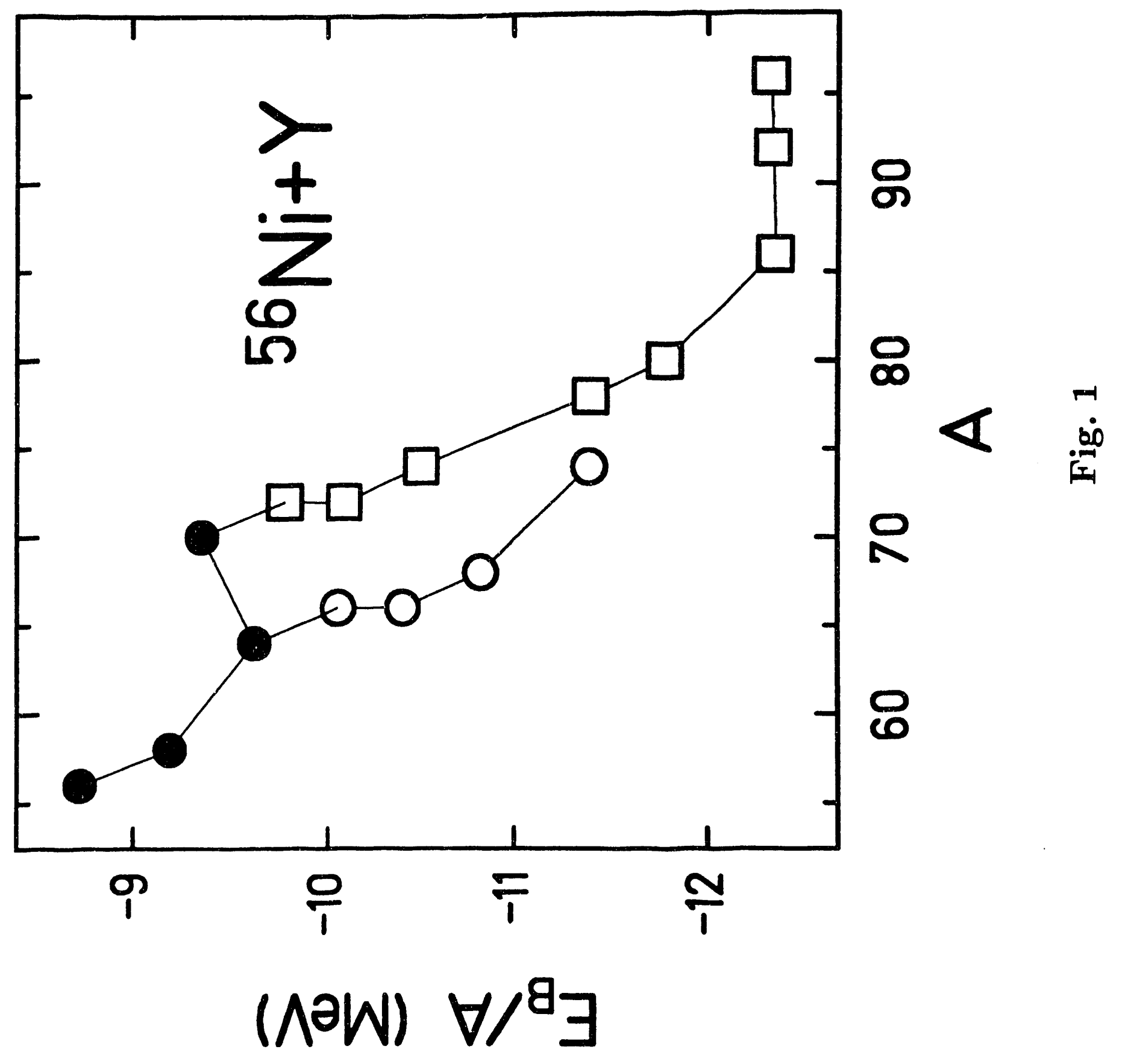




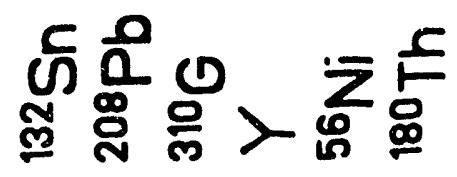

$$
\begin{aligned}
& \square \triangleleft \diamond \bullet \diamond *
\end{aligned}
$$

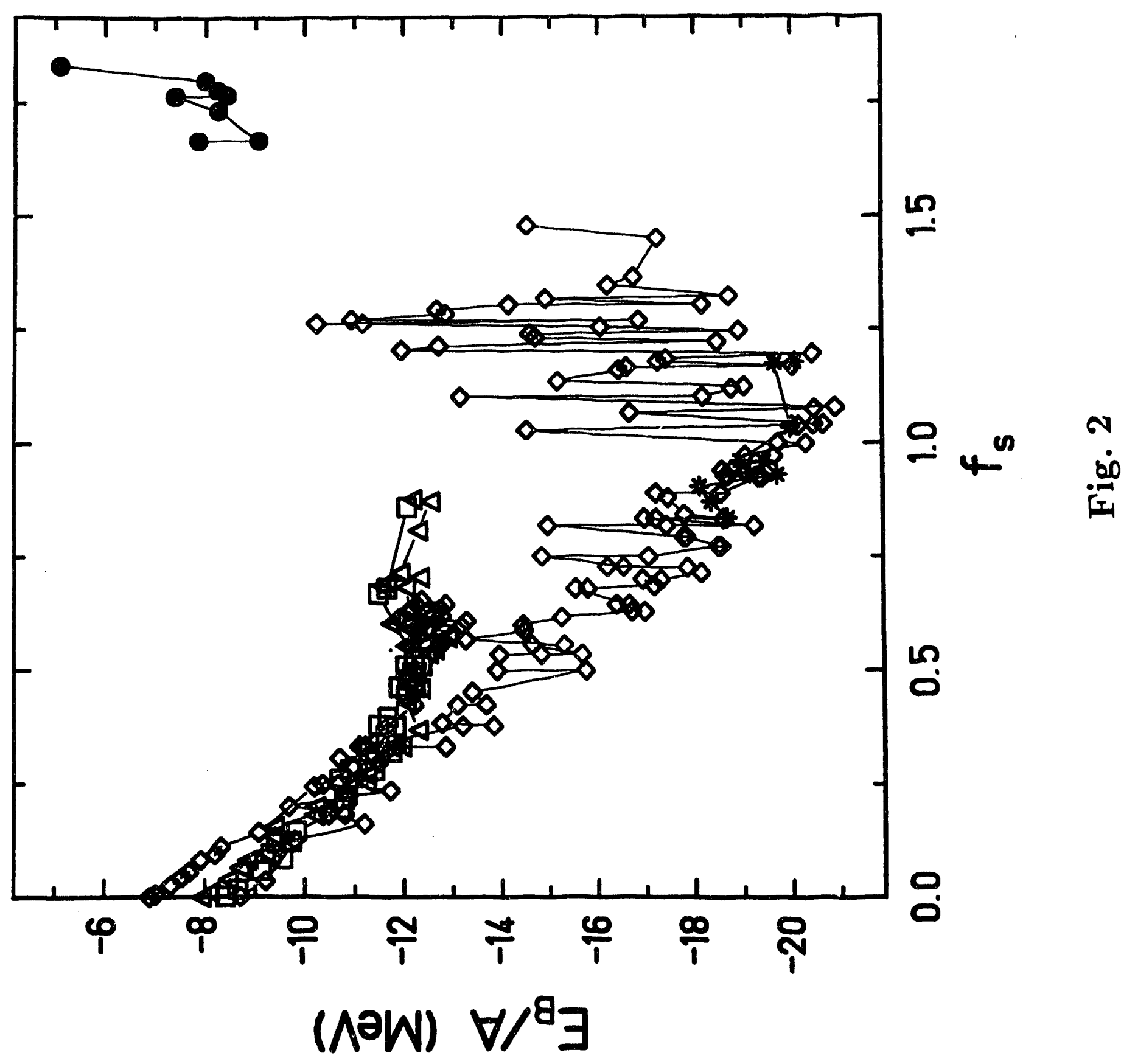




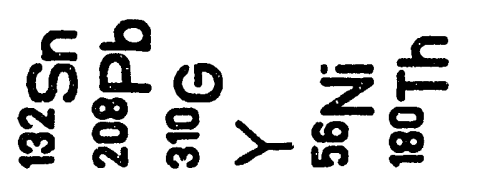

$\square \triangleleft \diamond \bullet \diamond *$

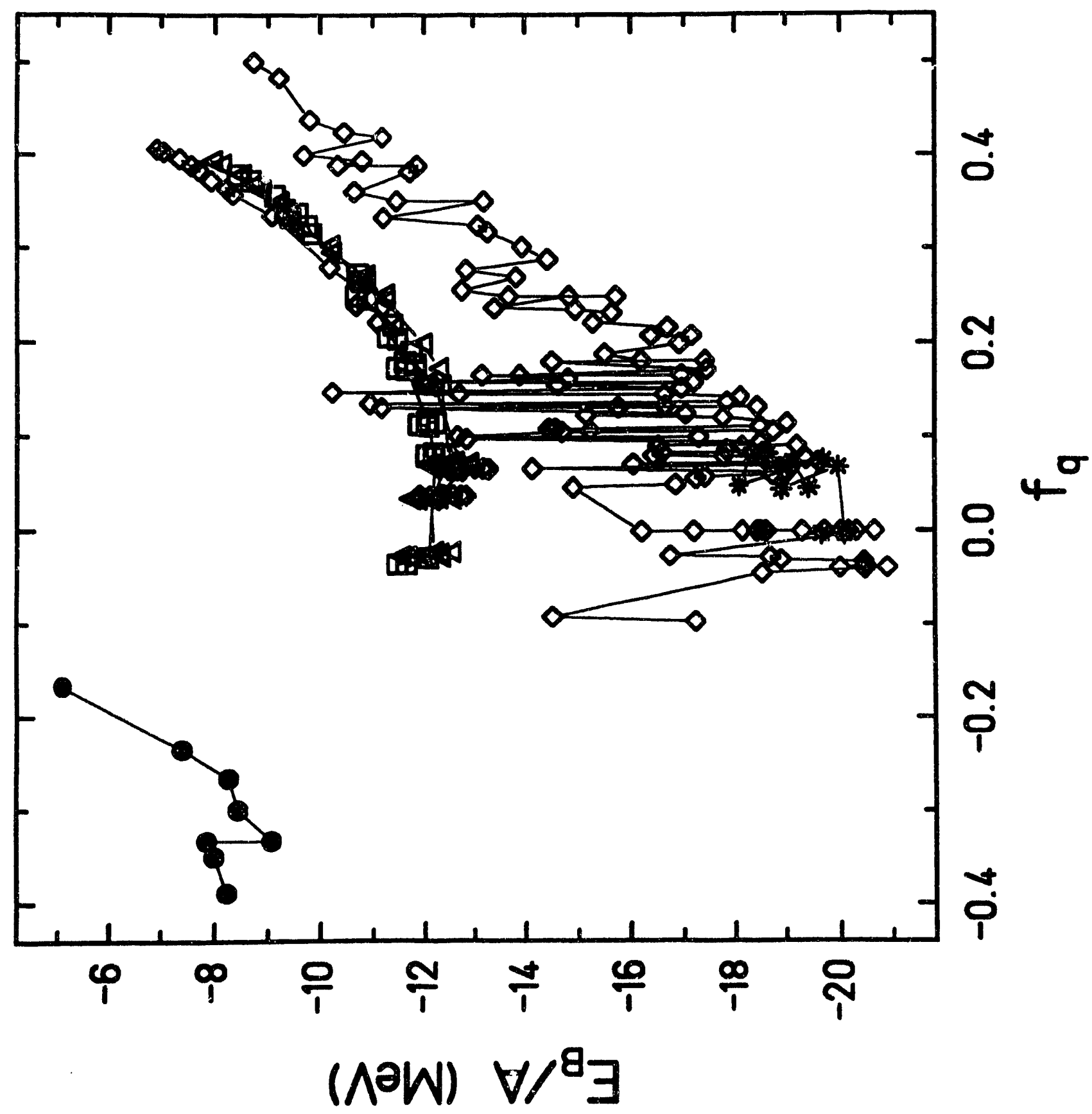

os 

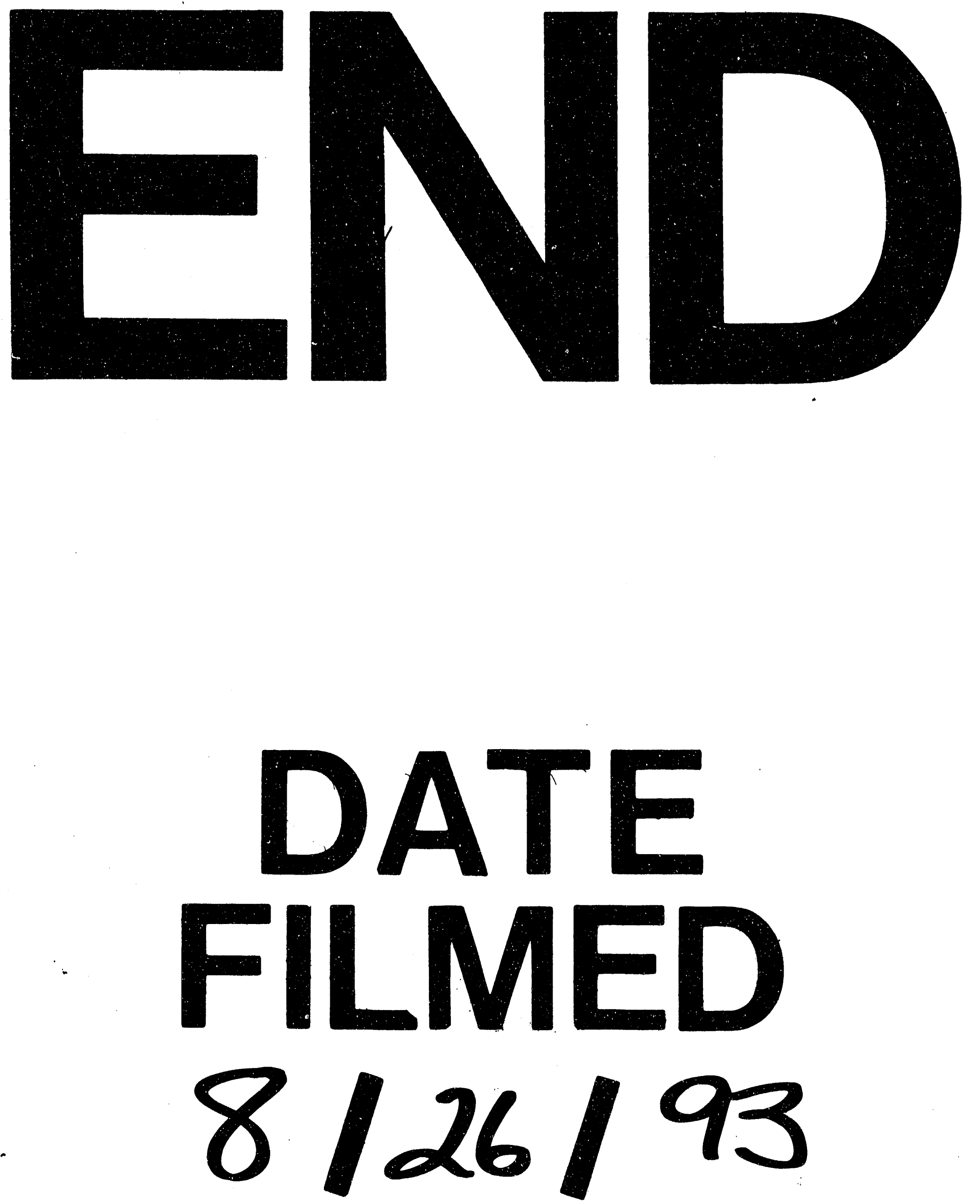
